\title{
Erratum di: Risposta degli Autori ad Allen et al.: "Un commento a Boyers et al.: 'Eltrombopag per il trattamento della porpora trombocitopenica immune o idiopatica cronica: valutazione di tecnologia singola presso il NICE,",
}

\author{
Dwayne Boyers • Xueli Jia • David Jenkinson • \\ Graham Mowatt
}

Pubblicato online: 5 marzo 2014

(C) Springer International Publishing Switzerland 2014

Erratum di: PharmacoEcon Ital Res Artic (2013) 15:157

DOI 10.1007/s40276-013-0017-Z

Questo articolo è una Traduzione dell'articolo: The Authors' Reply to Allen et al.: "A Comment on Boyers et al.: 'Eltrombopag for the Treatment of Chronic Immune or Idiopathic Thrombocytopenic Purpura: A NICE Single Technology Appraisal'". PharmacoEconomics 2013: 31(1):91. DOI 10.1007/s40273-012-0005-6.

La versione online dell' articolo originale può essere trovata al doi:10.1007/s40276-013-0017-z.

D. Boyers $(\varangle)$

Health Economics Research Unit, Institute of Applied Health

Sciences, University of Aberdeen, Foresterhill, Aberdeen AB25

2ZD, Scotland

e-mail: d.boyers@abdn.ac.uk

D. Boyers · X. Jia · D. Jenkinson · G. Mowatt

Health Services Research Unit, Institute di Applied Health

Sciences, University of Aberdeen, Aberdeen, Scotland 